\title{
Effects of individualized Tai-Chi on balance and lower-limb strength in older adults
}

\author{
I-Wen Penn ${ }^{1,2+}$, Wen-Hsu Sung ${ }^{3 \dagger}$, Chien-Hui Lin ${ }^{3}$, Eric Chuang ${ }^{4}$, Tien-Yow Chuang ${ }^{5}$ and Pei-Hsin Lin ${ }^{6 *}$
}

\begin{abstract}
Background: To investigate whether a simplified and personalized Tai-Chi program could be beneficial for practitioners. A prospective quasi-experimental observer-blinded controlled trial was done in Beitou District of Taipei City.

Methods: Community-dwelling adults aged 65 and older without debilitating disease $(N=50)$ participated the study. Those who were willing to participate in exercise program were assigned to individualized Tai-Chi (iTC) group $(n=20)$, receiving iTC training for 8 weeks, and traditional Tai-Chi (tTC) group $(n=15)$, receiving tTC training for 8 weeks. Those who were not willing to participate in exercise training were included in the control group $(n=15)$. Functional balance tests, the Berg Balance Scale (BBS), timed up-and-go (TUG) test, functional-reach test, and measurement of lowerextremity muscle strength were conducted before and 8 weeks after the intervention.

Results: Significant improvements were noted in all functional balance tests and strength assessments of 16 major lower-limb muscle groups in participants of the iTC group compared to the control group, whereas only BBS and muscle strength of hips and ankles were improved in the tTC group. Practitioners of iTC outperformed tTC in BBS and strength of two major muscles.

Conclusions: Personalized Tai-Chi training designed based on an objective measurement and conducted according to graded intensity and complexity benefitted practitioners after a short period.

Trial registration: Trial registration number: ClinicalTrials.gov ID: NCT03659396, Unique Protocol ID: 1000087

Date of registration: 03/28/2017

The trial was registered retrospectively
\end{abstract}

Keywords: Tai-chi, Exercise, Muscle strength, Functional balance

\section{Background}

Tai-Chi, characterized by a sequence of gentle, low-impact, and coordinated movements, is an appropriate form of exercise for older adults [1] because it involves minimal strain on joints and the cardiovascular system. In addition to the benefits from general physical activity, such as improving cardiopulmonary fitness and lowering blood pressure [2-5], Tai-Chi increases muscle strength in the lower extremities, improves balance control, proprioception, and postural adaption, and reduces the risk of falling in older adults [6-9].

Individuals of a similar biological age may exhibit a wide variance in their responses to exercise because of

\footnotetext{
* Correspondence: phlin2@vghtpe.gov.tw

${ }^{\dagger}$ I-Wen Penn and Wen-Hsu Sung contributed equally to this work.

${ }^{6}$ Center for Rehabilitation and Technical Aids, Taipei Veterans General

Hospital, Taipei 11217, Taiwan

Full list of author information is available at the end of the article
}

varying aging processes [1]. Thus, the prescription of Tai-Chi exercise should be personalized with regard to the intensity and difficulty, and exercise levels should gradually progress based on the functional ability of practitioners [10]. Moreover, the complexity and duration of traditional Tai-Chi (tTC) sequences may be too challenging for some older adults to execute [11, 12]. Therefore, we hypothesize that compared with a tTC program, an individualized Tai-Chi (iTC) program may be more effective in achieving the exercise's training goals, maximizing beneficial effects, and improving exercise adherence.

Several simplified Tai-Chi programs have been developed [11-14]. However, these exercises have primarily been designed in accordance with experts' opinions without considering participants' ability, and the training sessions have not involved graded progression because

(c) The Author(s). 2019 Open Access This article is distributed under the terms of the Creative Commons Attribution 4.0 International License (http://creativecommons.org/licenses/by/4.0/), which permits unrestricted use, distribution, and reproduction in any medium, provided you give appropriate credit to the original author(s) and the source, provide a link to the Creative Commons license, and indicate if changes were made. The Creative Commons Public Domain Dedication waiver (http://creativecommons.org/publicdomain/zero/1.0/) applies to the data made available in this article, unless otherwise stated. 
participants performed exercises at the same level of intensity and complexity throughout the program. We previously reported the development of a personalized Tai-Chi exercise program for older adults based on the measurements of practitioners' center of pressure displacement [15]. However, few participants were included, only the strength of the knee extensor was assessed, and a control group was not included; nevertheless, the findings of that study formed the basis of the present study for prescribing iTC moves according to participants' balance abilities and executing exercises in a graded manner as practitioners improved their balance control $[15,16]$. In the present study, we investigated the effect of an iTC regimen on functional balance control and lower-extremity muscle strength and compared the results of the iTC exercise group with those of control and tTC exercise groups.

\section{Methods}

This study was a prospective quasi-experimental observerblinded controlled trial. Participants received baseline assessments and were followed up after 8 weeks. The study was approved by the Institutional Review Board of National Yang-Ming University \#1000087. Written informed consent was obtained from all participants.

\section{Participants}

This is a quasi-experimental observer-blinded controlled trial study. Participants were recruited in Beitou District of Taipei City. As shown in Fig. 1, 70 individuals were initially recruited to the study. Those who were unwilling to practice Tai-Chi were assigned to the control group $(n=24)$, while those joined the Tai-Chi group were free to choose between joining either iTC $(n=25)$ or tTC $(n=21)$ group to enhance their stays to the program. All participants received education program that contained health information related to exercise, nutrition, fall, and balance. Participants of the iTC and tTC groups were asked to attend 24 Tai-Chi exercise classes, 3 classes per week, 30 min per class for 8 weeks. Some of them, however, failed to complete all 24 classes due to the reasons listed in Fig. 1; their data were not included in the analyses. The post-intervention evaluation was performed in 20,15, and 15 individuals of the iTC, tTC, and control group, respectively (Fig. 1).

\section{Inclusion criteria}

The inclusion criteria for the study were as follows: (a) aged 65 or older; (b) can understand and follow simple verbal instructions to perform exercises; (c) can stand independently; (d) can walk independently (with or

Individuals willing to participate the study

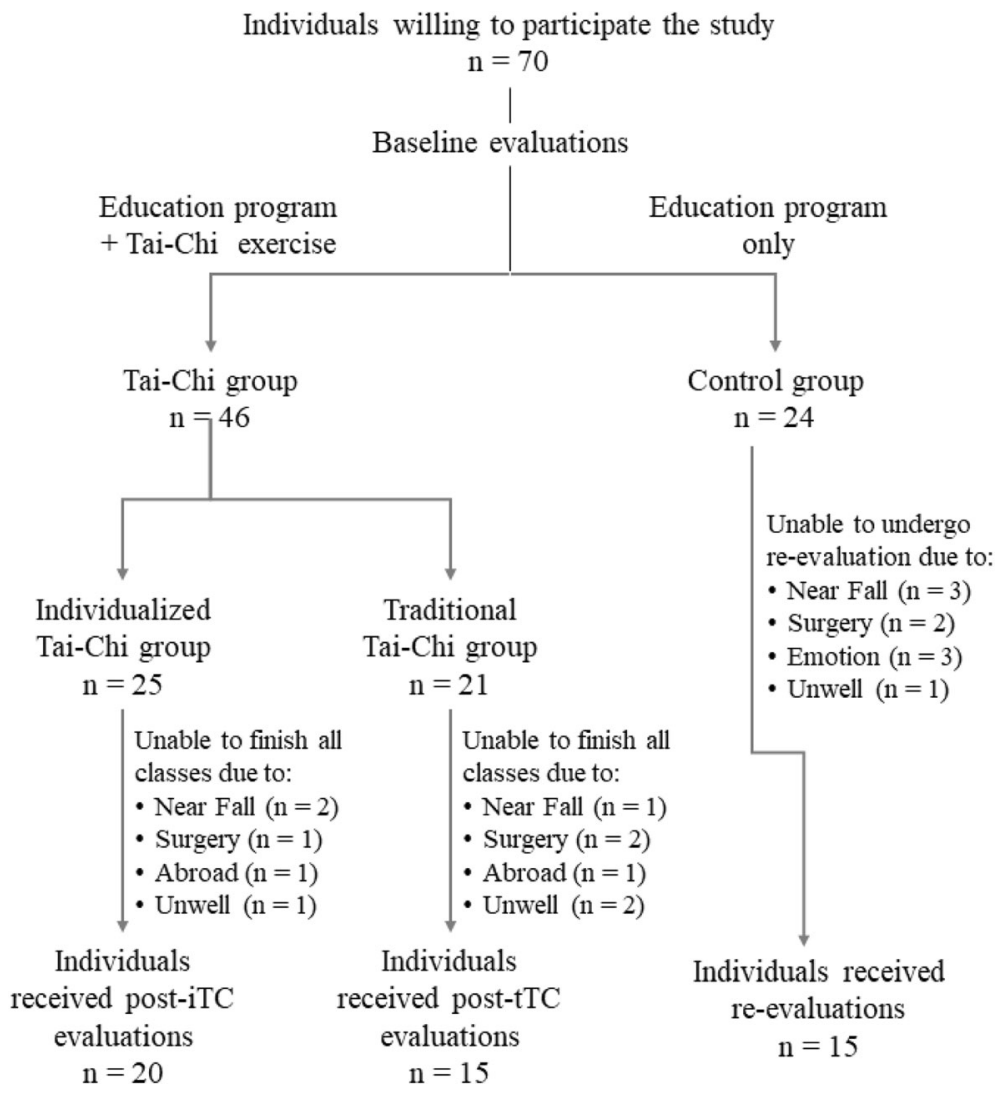

Fig. 1 Flowchart explaining assignment of the participants to the study and control groups 
without assistive devices); and (e) can stand up from a chair independently (with or without assistive devices). The evaluation was performed by a certified physical therapist.

\section{Exclusion criteria}

The exclusion criteria were as follows: (a) presence of any disorders of the central nervous system; (b) any orthopedic or cardiovascular disorders impeding ambulatory function or the ability to stand; (c) severe hearing impairment that inhibits ability to follow evaluation instructions and therapist guidance; (d) severe visual impairment that influences the individual's safety during execution of exercises; (e) participation in other similar trials; (f) any lower-limb fractures in the preceding 6 months; and (g) prior experience in Tai-Chi exercise for more than 2 months.

\section{Assignment and blinding}

Community-dwelling individuals who joined the study but were unwilling to practice Tai-Chi was recruited to the control group and received only the education program. Those who were willing to perform Tai-Chi exercises in addition to the education program could choose to join either iTC exercise or tTC exercise group. The current study is a forerunner to a randomized controlled trial. The pre- and post-intervention evaluations were performed by physical therapists who were blinded to the exercise treatments.

\section{Individualized tai-chi exercise group}

We first evaluated participants' COP displacement by using a computerized evaluation system that consisted of a computer, a force platform measuring an area of $100 \times 100 \mathrm{~cm} 2$, and strain gauges attached to load cells located at the four corners of the force plate. Strain gauges acquired a signal input for COP displacement. The classical 24-form Yang-style Tai-Chi exercise were classified into sixteen foot-support patterns, including four spatial directions (anterior, posterior, right, and left) at four feet positions (parallel stance, standing with the feet shoulder-width apart, left foot forward, and right foot forward), and four difficulty levels. At each feet-position, participants were asked to lean forward, backward, to the left, and to the right as far as possible at a comfortable, self-selected pace and maintain balance for $5 \mathrm{~s}$. We divided all Tai-Chi moves into four difficulty levels by using normalized COP displacement at anteroposterior and mediolateral directions: moves with COP displacement $<25,25-50 \%, 50-75 \%$, and $>75 \%$ of normalized values were classified as level 1,2, 3 and 4, respectively [15]. Based on the results of the maximum COP displacement and individual endurance, participants were assigned 3-5 moves at an appropriate difficulty level in each session. Participants repeated these selected moves for $30 \mathrm{~min}$ in each training session, three sessions per week for 8 weeks. The exercise program commenced with a low-difficulty level move and then progressed to higher difficulty levels as participants became adept at each move [17].

\section{Traditional tai-chi exercise group}

Participants in this program learned and practiced a classical 24-form Yang-style Tai-Chi exercise [18] under the instruction of certified Tai-Chi masters. The exercise program consisted of three sessions per week for 8 weeks. The duration of each session was $30 \mathrm{~min}$. For each session, participants usually practiced a complete Yang-Style Tai-Chi exercise two or three times.

\section{The control group}

Participants who were unwilling to participate in the exercise program were included as the control group. After the baseline assessments, participants attended education classes and received information related to nutrition, exercise, fall, and balance. The follow-up assessments were performed 8 weeks later; those failed to attend such evaluations due to the reasons listed in Fig. 1 were excluded from the study.

\section{Outcome measurements}

Functional balance tests, including Berg Balance Scale (BBS), timed up-and-go (TUG) test, functional-reach test, and measurement of lower-extremity muscle strength were conducted before and 8 weeks after the intervention by a certified physical therapist who was blinded to the treatment allocation. The BBS test consisted of 14 items that evaluated individuals' balance in sitting, standing, and changing positions. Scoring was determined using an ordinal 5-point scale with a total score range from 0 to 56 , where higher scores indicated superior balance [19].

The TUG test is a simple test used to assess a person's mobility and requires both static and dynamic balance [20]. The test measures the time taken by participants to stand up from a chair, walk $3 \mathrm{~m}$ at a comfortable speed, turn around, return to the chair, and then sit down. Three timed trials are needed to ensure performance stability in the TUG test. Therefore, mean values for three trials, with 1-min rest between each trial, were used for analysis.

The functional-reach test was used to assess dynamic balance; this test measured the maximal distance participants could reach forward beyond the length of their own arms. Participants were asked to stand next to a wall and reach forward as far as possible without moving their feet. The additional reaching distance was then recorded in centimeters [21]. 
Lower-extremity muscle strength was measured using a hand-held isometric dynamometer (Micro $\mathrm{FET}^{\circ} 3$, Hoggan Health Industries). The portable digital dynamometer performs accurate, objective muscle testing and enables assessors to stabilize and assist individuals while keeping one hand free. In this study, the following lower-limb muscles were bilaterally measured: hip flexor, hip extensor, hip abductor, hip adductor, knee extensor, knee flexor, ankle dorsiflexor, and ankle plantar flexor. The participants were asked to perform maximal isometric contractions for at least $3 \mathrm{~s}$.

\section{Statistical analysis}

A one-way ANOVA was conducted to compare the three groups with respect to their baseline characteristics, such as age, weight, height, and lower-limb muscle strength. Paired t-tests were conducted to compare the baseline and follow-up assessments in each group. A two-way repeated measures ANOVA was conducted to test the effect of interaction between time and group to compare the baseline and follow-up assessment of forward-reach distance, up-and-go time, and lower-limb muscle strength. To further explain the interaction, the simple effect of time at each group was also tested. All statistical analyses were performed using STATA version 14.2 (College Station, TX). Statistical significance was defined as a $p$ value of $<0.05$.

\section{Results}

\section{Demographic characteristics of participants in the three groups}

The sample size determined using G*Power 3.1.9.4 software was based on the results of our pilot study (Sung et al., 2018). With $80 \%$ power assuming an effect size of 0.5 at a two-tailed significance level of 0.05 , the calculated sample size was at least 14 participants in each group. We initially recruited 70 participants, of whom 46 received the education program along with Tai-Chi courses and 24 received only the education program. The flowchart of the study is presented in the additional file (Fig. 1). Participants of both Tai-Chi groups were required to complete all 24 exercise sessions ( $30 \mathrm{~min}$ each), which were performed three times a week for 8 weeks. All classes were supervised by the study controllers and at least one of the five Tai-Chi masters. Of the 25, 21, and 24 participants assigned to $\mathrm{iTC}$, tTC, and control groups, 5, 6, and 9 individuals could not finish the program, respectively. Finally, a total of 50 participants underwent the post-intervention evaluation. The demographic characteristics of participants who completed the study are listed in Table 1. No significant difference was observed in the distribution of age, sex, height, and weight among the iTC, tTC, and control groups.
Table 1 Demographic characteristics of participants

\begin{tabular}{lllll}
\hline & $\begin{array}{l}\text { Individualized } \\
\text { Tai-Chi Group } \\
(n=20)\end{array}$ & $\begin{array}{l}\text { Traditional } \\
\text { Tai-Chi Group } \\
(n=15)\end{array}$ & $\begin{array}{l}\text { Control } \\
\text { Group } \\
(n=15)\end{array}$ & $p$ value \\
\hline $\begin{array}{l}\text { Age (y) } \\
\text { Sex }\end{array}$ & $76.45 \pm 8.63$ & $75.27 \pm 5.20$ & $73.4 \pm 8.2$ & 0.515 \\
$\quad$ Male & $2(10 \%)$ & $2(13.3 \%)$ & $3(20 \%)$ & 0.872 \\
Female & $18(90 \%)$ & $13(86.6 \%)$ & $12(80 \%)$ & \\
Ht (cm) & $153.57 \pm 7.88$ & $155.8 \pm 6.6$ & $154.67 \pm 6.66$ & 0.667 \\
Wt (kg) & $53.70 \pm 8.30$ & $57.9 \pm 7.87$ & $52.73 \pm 6.24$ & 0.125 \\
\hline Abbreviations: $y$ years, Ht Height, Wt Weight & &
\end{tabular}

No significant difference observed between the baseline assessment results of the three groups

Table 2 presents the results of participants in the iTC, tTC, and control group for the baseline assessments, which consisted of three functional balance tests and a lower-limb muscle strength assessment. No significant difference was observed among the three groups in the

Table 2 Baseline assessments of participants

\begin{tabular}{|c|c|c|c|c|}
\hline & $\begin{array}{l}\text { Individualized } \\
\text { Tai-Chi } \\
\text { Group } \\
(n=20)\end{array}$ & $\begin{array}{l}\text { Complete } \\
\text { Tai-Chi } \\
\text { Group } \\
(n=15)\end{array}$ & $\begin{array}{l}\text { Control } \\
\text { Group } \\
(n=15)\end{array}$ & $p$ value \\
\hline \multicolumn{5}{|c|}{ Functional Balance Assessment } \\
\hline BBS & $45.05 \pm 12.17$ & $49.33 \pm 4.52$ & $45.13 \pm 12.35$ & 0.944 \\
\hline TUG (seconds) & $12.00 \pm 8.45$ & $9.10 \pm 1.94$ & $11.24 \pm 9.59$ & 0.527 \\
\hline $\mathrm{FRD}(\mathrm{cm})$ & $24.30 \pm 7.40$ & $23.75 \pm 6.47$ & $21.66 \pm 7.01$ & 0.531 \\
\hline \multicolumn{5}{|l|}{ Muscle Strength } \\
\hline $\mathrm{RH} F$ & $14.55 \pm 3.71$ & $16.39 \pm 4.32$ & $13.64 \pm 5.87$ & 0.261 \\
\hline LHF & $14.47 \pm 3.32$ & $15.29 \pm 4.72$ & $13.09 \pm 5.00$ & 0.373 \\
\hline $\mathrm{RH} \mathrm{E}$ & $13.64 \pm 4.91$ & $16.06 \pm 3.33$ & $12.67 \pm 5.37$ & 0.129 \\
\hline LHE & $14.36 \pm 5.49$ & $15.71 \pm 3.46$ & $12.57 \pm 4.58$ & 0.194 \\
\hline $\mathrm{RH} A b$ & $12.67 \pm 3.66$ & $15.51 \pm 3.73$ & $12.04 \pm 2.65$ & $0.016^{*}$ \\
\hline $\mathrm{LH} A b$ & $12.57 \pm 3.20$ & $12.26 \pm 2.07$ & $12.31 \pm 3.08$ & 0.942 \\
\hline $\mathrm{RH} \mathrm{Ad}$ & $10.23 \pm 2.86$ & $11.07 \pm 2.09$ & $9.38 \pm 3.02$ & 0.240 \\
\hline $\mathrm{LH} A d$ & $9.67 \pm 2.63$ & $11.88 \pm 2.91$ & $9.74 \pm 3.12$ & 0.057 \\
\hline RK F & $11.20 \pm 2.83$ & $13.13 \pm 2.84$ & $10.09 \pm 3.31$ & $0.025^{*}$ \\
\hline LKF & $10.64 \pm 2.92$ & $12.81 \pm 2.69$ & $10.16 \pm 3.99$ & 0.061 \\
\hline RK E & $15.93 \pm 4.89$ & $18.06 \pm 3.92$ & $15.15 \pm 6.48$ & 0.286 \\
\hline LKE & $15.58 \pm 5.46$ & $16.91 \pm 3.08$ & $13.95 \pm 5.06$ & 0.240 \\
\hline RA Df & $11.10 \pm 2.85$ & $11.87 \pm 3.01$ & $10.57 \pm 2.80$ & 0.466 \\
\hline LA Df & $11.32 \pm 2.65$ & $12.01 \pm 2.81$ & $10.85 \pm 2.43$ & 0.484 \\
\hline RA Pf & $15.41 \pm 3.71$ & $17.12 \pm 2.46$ & $16.06 \pm 5.10$ & 0.441 \\
\hline LA Pf & $14.88 \pm 3.96$ & $16.53 \pm 2.90$ & $15.51 \pm 4.85$ & 0.484 \\
\hline
\end{tabular}

Abbreviations: BBS Berg Balance Scale, TUG Timed up-and-go, FRD Forward reach distance, $R H$ Righ hip, F Flexor, $L H$ Left hip, $E$ Extensor, $A b$ Abductor, Ad Adductor, RK Right knee, LK Left knee, RA Right ankle, Df Dorsi-flexor, LA Left ankle, Pf Plantar-flexor ${ }^{*} p<0.05$ 
functional balance assessments. For muscle-strength measurements, the tTC group only exhibited higher scores for the right hip abductor $(p=0.017)$ and right knee flexor $(p=0.025)$ compared with iTC and control groups.

\section{Functional balance and muscle strength improved after tai-chi interventions}

As shown in Table 3, participants in the iTC group exhibited a significant improvement in three types of functional balance tests and all 16 groups of lower-limb muscle strength $(p<0.001-0.007)$. In the tTC group, only the BBS score $(p=0.005)$ and muscle strength of the bilateral hip flexors, right hip extensor, left hip abductor, right hip adductor, and bilateral ankle dorsiflexor improved significantly $(p=0.010-0.033)$. The control group also exhibited improved muscle strength of the right hip extensor $(p=0.033)$. The control group received health information regarding exercises and prevention of falls and underwent the same functional balance tests as did the other groups; they were also informed about the test results. This education program might have increased their awareness of exercise and encouraged self-training.

\section{Participants benefitted more from individualized tai-chi than from traditional tai-chi}

Table 4 presents the comparison of the results for the three groups based on 2-way repeated measures ANOVA. The interaction between time and group showed statistical significance over BBS, muscle strength over left hip abductor, right hip adductor, left hip adductor, right knee extensor, left ankle dorsi-flexor, right ankle plantar flexor and left ankle plantar flexor. Further analysis of the simple effect of time at each group showed no significant difference between baseline and follow-up assessment of all the measurements in the control group. By contrast, in the iTC group, there were significant differences between baseline and follow-up assessments of all the measurements except forward-reach distance. In the tTC group, some of the assessments showed significant differences between baseline and follow-up assessment. They were BBS, muscle strength over right hip flexor, right hip extensor, left hip abductor, right

Table 3 Baseline and follow-up assessment results of participants

\begin{tabular}{|c|c|c|c|c|c|c|c|c|c|}
\hline \multicolumn{4}{|c|}{ Individualized Tai-Chi Group $(n=20)$} & \multicolumn{3}{|c|}{ Traditional Tai-Chi Group $(n=15)$} & \multicolumn{3}{|c|}{ Control Group $(n=15)$} \\
\hline & Baseline & Follow-up & $p$ value & Baseline & Follow-up & $p$ value & Baseline & Follow up & $p$ value \\
\hline \multicolumn{10}{|c|}{ Functional Balance } \\
\hline BBS & $45.05 \pm 12.17$ & $48.00 \pm 11.35$ & $0.000^{*}$ & $49.33 \pm 4.52$ & $51.33 \pm 4.79$ & $0.000^{*}$ & $45.13 \pm 12.35$ & $45.87 \pm 12.36$ & 0.098 \\
\hline TUG & $12.00 \pm 8.45$ & $10.10 \pm 7.65$ & $0.000^{*}$ & $9.10 \pm 1.94$ & $8.33 \pm 1.71$ & 0.050 & $11.24 \pm 9.59$ & $10.52 \pm 8.86$ & 0.075 \\
\hline FRD & $24.30 \pm 7.40$ & $26.24 \pm 6.82$ & $0.005^{*}$ & $23.75 \pm 6.47$ & $25.52 \pm 4.11$ & 0.299 & $21.66 \pm 7.01$ & $22.03 \pm 6.65$ & 0.728 \\
\hline \multicolumn{10}{|c|}{ Muscle Strength } \\
\hline $\mathrm{RH} F$ & $14.55 \pm 3.71$ & $17.71 \pm 3.84$ & $0.002^{*}$ & $16.39 \pm 4.32$ & $18.88 \pm 3.25$ & $0.011^{*}$ & $13.64 \pm 5.87$ & $14.48 \pm 4.57$ & 0.340 \\
\hline LHF & $14.47 \pm 3.32$ & $16.98 \pm 3.86$ & $0.005^{*}$ & $15.29 \pm 4.72$ & $17.90 \pm 3.75$ & $0.010^{*}$ & $13.09 \pm 5.00$ & $13.89 \pm 4.45$ & 0.393 \\
\hline $\mathrm{RH} E$ & $13.64 \pm 4.91$ & $17.31 \pm 5.60$ & $0.000^{*}$ & $16.06 \pm 3.33$ & $18.10 \pm 3.85$ & $0.026^{*}$ & $12.67 \pm 5.37$ & $14.65 \pm 5.00$ & $0.033^{*}$ \\
\hline LHE & $14.36 \pm 5.49$ & $16.98 \pm 3.86$ & $0.001^{*}$ & $15.71 \pm 3.46$ & $17.90 \pm 3.75$ & 0.083 & $12.57 \pm 4.58$ & $13.89 \pm 4.45$ & 0.128 \\
\hline $\mathrm{RH} A b$ & $12.67 \pm 3.66$ & $15.52 \pm 4.72$ & $0.004^{*}$ & $15.51 \pm 3.73$ & $16.94 \pm 2.54$ & 0.112 & $12.04 \pm 2.65$ & $13.09 \pm 2.77$ & 0.088 \\
\hline $\mathrm{LH} A b$ & $12.57 \pm 3.20$ & $14.90 \pm 4.21$ & $0.007^{*}$ & $12.26 \pm 2.07$ & $16.00 \pm 2.87$ & $0.000^{*}$ & $12.31 \pm 3.08$ & $12.01 \pm 2.54$ & 0.623 \\
\hline $\mathrm{RH}$ Ad & $10.23 \pm 2.86$ & $13.06 \pm 4.20$ & $0.002^{*}$ & $11.07 \pm 2.09$ & $12.92 \pm 2.99$ & $0.033^{*}$ & $9.38 \pm 3.02$ & $9.61 \pm 3.21$ & 0.638 \\
\hline LH Ad & $9.67 \pm 2.63$ & $12.58 \pm 3.59$ & $0.000^{*}$ & $11.88 \pm 2.91$ & $13.53 \pm 2.68$ & 0.054 & $9.74 \pm 3.12$ & $10.20 \pm 2.36$ & 0.308 \\
\hline RK F & $11.20 \pm 2.83$ & $13.86 \pm 3.87$ & $0.000^{*}$ & $13.13 \pm 2.84$ & $14.52 \pm 2.70$ & 0.096 & $10.09 \pm 3.31$ & $10.61 \pm 2.73$ & 0.367 \\
\hline LKF & $10.64 \pm 2.92$ & $13.53 \pm 4.59$ & $0.001^{*}$ & $12.81 \pm 2.69$ & $14.15 \pm 2.94$ & 0.090 & $10.16 \pm 3.99$ & $10.73 \pm 2.85$ & 0.435 \\
\hline RK E & $15.93 \pm 4.89$ & $20.47 \pm 5.81$ & $0.000^{*}$ & $18.06 \pm 3.92$ & $19.06 \pm 4.40$ & 0.220 & $15.15 \pm 6.48$ & $15.24 \pm 4.77$ & 0.936 \\
\hline LKE & $15.58 \pm 5.46$ & $18.19 \pm 6.11$ & $0.007^{*}$ & $16.91 \pm 3.08$ & $18.21 \pm 3.66$ & 0.154 & $13.95 \pm 5.06$ & $14.20 \pm 3.82$ & 0.793 \\
\hline RA Df & $11.10 \pm 2.85$ & $13.88 \pm 3.43$ & $0.001^{*}$ & $11.87 \pm 3.01$ & $14.00 \pm 2.80$ & $0.010^{*}$ & $10.57 \pm 2.80$ & $11.81 \pm 2.69$ & 0.140 \\
\hline LA Df & $11.32 \pm 2.65$ & $14.26 \pm 3.40$ & $0.000^{*}$ & $12.01 \pm 2.81$ & $14.47 \pm 3.18$ & $0.003^{*}$ & $10.85 \pm 2.43$ & $11.52 \pm 2.78$ & 0.263 \\
\hline RA Pf & $15.41 \pm 3.71$ & $20.46 \pm 6.74$ & $0.000^{*}$ & $17.12 \pm 2.46$ & $19.09 \pm 3.66$ & 0.068 & $16.06 \pm 5.10$ & $17.09 \pm 4.64$ & 0.280 \\
\hline LA Pf & $14.88 \pm 3.96$ & $20.27 \pm 6.53$ & $0.000^{*}$ & $16.53 \pm 2.90$ & $17.44 \pm 3.90$ & 0.220 & $15.51 \pm 4.85$ & $15.57 \pm 4.61$ & 0.936 \\
\hline
\end{tabular}

Abbreviations: BBS Berg Balance Scale, TUG Timed up-and-go, FRD Forward reach distance, $R H$ Righ hip, $F$ Flexor, $L H$ Left hip, $E$ Extensor, $A b$ Abductor, $A d$ Adductor, $R K$ Right knee, LK Left knee, RA Right ankle, Df Dorsi-flexor, LA Left ankle, Pf Plantar-flexor ${ }^{*} p<0.05$ 
Table 4 Comparisons among Individualized Tai-Chi, Traditional Tia-Chi and Control group using Two-way Repeated Measures ANOVA

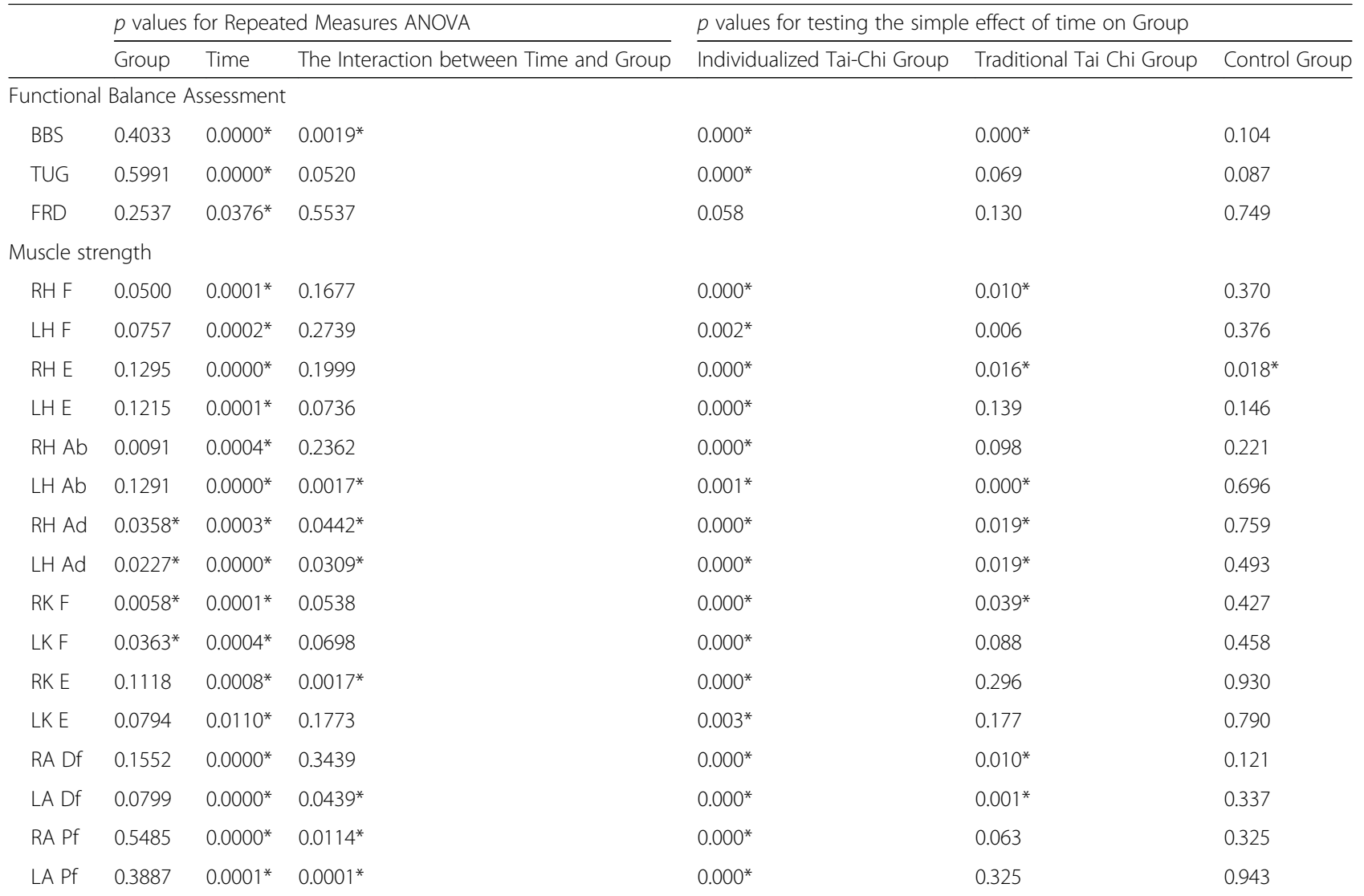

Abbreviations: BBS Berg Balance Scale, TUG Timed up-and-go, FRD Forward reach distance, $R H$ Righ hip, $F$ Flexor, $L H$ Left hip, $E$ Extensor, $A b$ Abductor, $A d$ Adductor, $R K$ Right knee, $L K$ Left knee, RA Right ankle, Df Dorsi-flexor, LA Left ankle, Pf Plantar-flexor

${ }^{*} p<0.05$

hip adductor, left hip adductor, right knee flexor, right ankle dorsi-flexor and left ankle dorsi-flexor.

\section{Discussion}

Tai-Chi is based on continuous fluid movements involving a semi-squat posture that enables an individual's bodyweight to be shifted from side to side [22, 23]. Concentric and eccentric muscle contractions performed in Tai-Chi can increase lower-limb muscle strength [7, 22]. One previous study focused on the beneficial effect of Tai-Chi training on the muscle strength of the knee extensors or flexors in older adults [24], whereas other studies have reported the effects of Tai-Chi training on the hip and ankle muscles [25-27]. Thus, a range of results have been reported due to the employment of different Tai-Chi moves, training durations, and assessment methods [28].

In this study, we found that although both iTC and tTC training were beneficial for practitioners compared with controls, the 8-week iTC training could improve balance and muscle strength of all lower limbs, whereas tTC appeared to improve only the score of the BBS and the strength of some hip and ankle muscles but not knee flexors and extensors, which is in contrast to the findings of some previous studies [8, 29-31]. This result may indicate one of the following. (a) The intervention duration was too short for each Tai-Chi move to affect the knee muscles. Additionally, training was affected by the semi-squat posture of Tai-Chi, which induces stress on the knee muscles $[23,24]$. (b) The full set of tTC exercises may have involved moves that were too difficult for the elderly participants. The amplitude of weight shifting, as estimated by the degree of COP displacements, differs among various Tai-Chi moves [32]. Individuals vary in their abilities to voluntarily shift their weight in various spatial directions and to briefly maintain stability in different positions; thus, not all older individuals are able to complete Tai-Chi programs. In this study, we noted that participants in the tTC group often omitted or skipped difficult moves or performed the steps at their own will. Therefore, elderly participants may require individual consideration when a Tai-Chi exercise program is being developed for them [33]. The present study revealed that the beneficial effects associated with iTC exercises were superior to those observed from a tTC program. The participants of the iTC group in this study were 
asked to repeatedly practice only 3-5 moves in each session; this format enabled them to meet the trainer's requirements and experience the beneficial effects of the training sequence. The moves of iTC program were assigned according to the individual's balance control, as assessed through COP measurements. The training program was designed to be progressive and continually challenge participants. This goal was achieved by gradually incorporating Tai-Chi moves of increasingly substantial COP displacements $[34,35]$. After the initial moves had been learned and mastered, the difficulty of the exercise routine was increased to the next level in a graded manner. We therefore concluded that the superior results observed in the iTC group may have been attributable to the tailored moves and graded intensity and complexity mode of practices.

Our results agree with those of other studies that have reported significant improvements in leg balance from Tai-Chi exercises. However, these results contradict those reported in studies conducted by Woo et al. [36], which indicated that Tai-Chi training alone is not sufficient for improving balance. This divergence may be a result of the intensity and duration of the Tai-Chi intervention as well as the fact that muscle strength is only one factor that influences balance. Most studies have reported that older adults often must practice for relatively long periods, typically from 12 to 48 weeks, to benefit from various forms of exercise, including Tai-Chi [8, 29-31]. Notably, a mere 8-week intervention as described in the present study, provided similar beneficial effects as those reported in longer interventions. If the participants begin training at an intensity and complexity suited to them then they can more precisely perform sequences designed to improve muscle strength, neuromuscular control, proprioception, postural adaption, and balance $[8,25]$. Although balance abilities associated with the knee extensor and flexor are primary contributors to balance control, our results did not indicate a relationship between the knee extensor and flexor and improvements in BBS in tTC. Tai-Chi is regarded as an effective means of enhancing collective motor functions; the movements stimulate part of the brain that governs balance, thereby increasing overall steadiness [37-39].

The primary limitation of the present study was its quasi-experimental design. The findings of our exploratory analysis revealed that participants who were unwilling to join the exercise group tended to be older than participants who were willing to join the exercise group. Furthermore, the single-blind design used in this study may have caused a placebo effect, and the study involved only individual measurements of muscle power of each lower limb instead of a summary measure.

\section{Conclusions}

We found that the personalized Tai-Chi training program designed on the basis of an objective measurement and conducted according to graded intensity and complexity was more beneficial to practitioners after a short period of 8 weeks compared with the traditional full-course Tai-Chi training program.

\section{Abbreviations \\ BBS: Berg Balance Scale; COP: Center of pressure; Ht: Height; ITC: individualized Tai-Chi; LOS: Limit of stability; tTC: traditional Tai-Chi; TUG: Timed up-and-go; Wt: Weight; y: year}

\section{Acknowledgements}

The authors would like to express their gratitude to the Department of Physical Therapy and Assistive Technology, National Yang-Ming University, for the wonderful support during the research.

\section{Authors' contributions}

IWP, WHS, and PHL conceptualized and developed the methodologies used in this study. WHS and PHL developed the software. Formal analysis and data curation were performed by IWP, WHS, CHL, EC, TYC, and PHL. Writing of the manuscript, including the original draft preparation, review and editing, and visualization were performed by IWP, WHS, CHL, EC, TYC, and PHL. All authors read and approved the final manuscript.

\section{Funding}

This work was supported by grant NSC 100-2410-H-010-010-MY3 from the Ministry of Science and Technology, Taiwan, awarded to WHS. The funding agency approved the design of the study but had no role in the collection, analysis, and interpretation of the data, or in writing the manuscript.

\section{Availability of data and materials}

Requests for data and associated analyses with individuals' IDs removed or appropriately concealed can be addressed to Dr. Wen-Hsu Sung of NYMU.

\section{Ethics approval and consent to participate}

The Institutional Review Board of National Yang-Ming University approved this study (IRB number: 1000087). Written informed consent was obtained from all participants.

\section{Consent for publication \\ Not applicable.}

\section{Competing interests}

The authors declare that they have no competing interests.

\section{Author details}

${ }^{1}$ School of Medicine, Fu Jen Catholic University, New Taipei City 24205,

Taiwan. ${ }^{2}$ Department of Physical Medicine and Rehabilitation, Fu Jen Catholic University Hospital, Fu Jen Catholic University, New Taipei City 24352, Taiwan. ${ }^{3}$ Department of Physical Therapy and Assistive Technology, National Yang-Ming University, Taipei 11221, Taiwan. ${ }^{4}$ Department of Integrative Biology Alumnus, University of California, Berkeley, CA 94720, USA. ${ }^{5}$ Department of Physical Medicine \& Rehabilitation, Taipei Veterans General Hospital and National Yang Ming University, Taipei 11217, Taiwan. ${ }^{6}$ Center for Rehabilitation and Technical Aids, Taipei Veterans General Hospital, Taipei 11217, Taiwan.

Received: 14 February 2019 Accepted: 15 August 2019

Published online: 27 August 2019

\section{References}

1. Rdejlgm M. Exercise prescription for healthy populations with special consideration. In: Medicine" ACoS, editor. ACSMs guidelins for exercise testing and prescription. 10th ed. Philadelphia: Wolters Kluwer/Lippincott Williams \& Wilkins; 2018. p. 180-202.

2. Lan C, Lai JS, Chen SY, Wong MK. 12-month tai chi training in the elderly: its effect on health fitness. Med Sci Sports Exerc. 1998;30:345-51. 
3. Smith LL, Wherry SJ, Larkey LK, Ainsworth BE, Swan PD. Energy expenditure and cardiovascular responses to tai chi easy. Complement Ther Med. 2015; 23:802-5.

4. Young DR, Appel $L$, Jee S, Miller ER 3rd. The effects of aerobic exercise and T'ai chi on blood pressure in older people: results of a randomized trial. J Am Geriatr Soc. 1999;47:277-84.

5. Lee YM. The effects of tai chi on waist circumference and blood pressure in the elderly. J Phys Ther Sci. 2017;29:172-5.

6. Tsang WW, Hui-Chan CW. Effect of 4- and 8-wk intensive tai chi training on balance control in the elderly. Med Sci Sports Exerc. 2004;36:648-57.

7. Song QH, Zhang QH, Xu RM, Ma M, Zhao XP, Shen GQ, et al. Effect of taichi exercise on lower limb muscle strength, bone mineral density and balance function of elderly women. Int J Clin Exp Med. 2014;7:1569-76.

8. Huang ZG, Feng YH, Li YH, Lv CS. Systematic review and meta-analysis: tai chi for preventing falls in older adults. BMJ Open. 2017;7:e013661.

9. Jain A, Taylor J, Sanzo P, Zerpa C. The effect of tai chi on functional lower extremity mobility and strength, ankle proprioception, and postural adaptation in older adults. Am J Med Med Sci. 2017;7:229-37.

10. Zaleski AL, Taylor BA, Panza GA, Wu Y, Pescatello LS, Thompson PD, et al. Coming of age: considerations in the prescription of exercise for older adults. Methodist Debakey Cardiovasc J. 2016;12:98-104.

11. Li F, Fisher KJ, Harmer P, Shirai M. A simpler eight-form easy tai chi for elderly adults. J Aging Phys Act. 2003;11:206-18.

12. Wang H, Wei A, Lu Y, Yu B, Chen W, Lu Y, et al. Simplified tai chi program training versus traditional tai chi on the functional movement screening in older adults. Evid Based Complement Alternat Med. 2016;2016:5867810.

13. Chen KM, Chen WT, Huang MF. Development of the simplified tai chi exercise program (STEP) for frail older adults. Complement Ther Med. 2006;14:200-6.

14. Tousignant M, Corriveau H, Roy PM, Desrosiers J, Dubuc N, Hebert R. Efficacy of supervised tai chi exercises versus conventional physical therapy exercises in fall prevention for frail older adults: a randomized controlled trial. Disabil Rehabil. 2013;35:1429-35.

15. Sung WH, Liu CC, Wei SH, Chuang LR, Chuang E, Wang KA, et al. Feasibility and outcome of an individualized tai chi program for improving balance and strength in the elderly: apilot study. NeuroRehabilitation. 2018;43:509-18.

16. Kim HD. Effects of tai chi exercise on the center of pressure trace during obstacle crossing in older adults who are at a risk of falling. J Phys Ther Sci. 2009:21:49-54.

17. Juras G, Slomka K, Fredyk A, Sobota G, Bacik B. Evaluation of the limits of stability (LOS) balance test. J Hum Kinet. 2008;19:39-52.

18. Zou Y. History of the 24 forms simplified Tai Chi routine/movements of 24 forms simplified Tai Chi routine. https://www.academia.edu/5974363/ History_of_the_24_Forms_Simplified_Tai_Chi_Routine_Movements_of_24_ Forms_Simplified_Tai_Chi_Routine. Accessed 24 Aug 2019.

19. Berg K, Wood-Dauphinee S, Williams JI. The balance scale: reliability assessment with elderly residents and patients with an acute stroke. Scand $J$ Rehabil Med. 1995:27:27-36.

20. Podsiadlo D, Richardson S. The timed "up \& go": a test of basic functional mobility for frail elderly persons. J Am Geriatr Soc. 1991;39:142-8.

21. Duncan PW, Weiner DK, Chandler J, Studenski S. Functional reach: a new clinical measure of balance. J Gerontol. 1990;45:M192-7.

22. Lan C, Lai JS, Chen SY, Wong MK. Tai chi Chuan to improve muscular strength and endurance in elderly individuals: a pilot study. Arch Phys Med Rehabil. 2000;81:604-7.

23. Pereira MM, Oliveiria RJ, Silva MAF, Souza LHR, Vianna LG. Effects of tai chi Chuan on knee extensor muscle strength and balance in elderly women. Res Bras Fisioter. 2008;12:121-6.

24. Ye J, Cai S, Zhong W, Cai S, Zheng Q. Effects of tai chi for patients with knee osteoarthritis: a systematic review. J Phys Ther Sci. 2014;26:1133-7.

25. Xu D, Hong Y, Li J, Chan K. Effect of tai chi exercise on proprioception of ankle and knee joints in old people. Br J Sports Med. 2004;38:50-4.

26. Li JX, Xu DQ, Hong Y. Effects of 16-week tai chi intervention on postural stability and proprioception of knee and ankle in older people. Age Ageing. 2008:37:575-8

27. Liu B, Liu ZH, Zhu HE, Mo JC, Cheng DH. Effects of tai chi on lower-limb myodynamia in the elderly people: a meta-analysis. J Tradit Chin Med. 2011; 31:141-6.

28. Hong Y, Li JX. Biomechanics of tai chi: a review. Sports Biomech. 2007;6:453-64.

29. Li F, Harmer P, Fitzgerald K, Eckstrom E, Stock R, Galver J, et al. Tai chi and postural stability in patients with Parkinson's disease. N Engl J Med. 2012; 366:511-9.
30. Taylor-Piliae RE, Hoke TM, Hepworth JT, Latt LD, Najafi B, Coull BM. Effect of tai chi on physical function, fall rates and quality of life among older stroke survivors. Arch Phys Med Rehabil. 2014;95:816-24.

31. Day L, Hill KD, Stathakis VZ, Flicker L, Segal L, Cicuttini F, et al. Impact of tai-chi on falls among preclinically disabled older people: a randomized controlled trial. J Am Med Dir Assoc. 2015;16:420-6.

32. Leung ES, Tsang WW. Comparison of the kinetic characteristics of standing and sitting tai chi forms. Disabil Rehabil. 2008;30:1891-900.

33. Lemay JF, Gagnon DH, Nadeau S, Grangeon M, Gauthier C, Duclos C. Center-of-pressure total trajectory length is a complementary measure to maximum excursion to better differentiate multidirectional standing limits of stability between individuals with incomplete spinal cord injury and able-bodied individuals. J Neuroeng Rehabil. 2014;11:8.

34. Szturm T, Fallang B. Effects of varying acceleration of platform translation and toes-up rotations on the pattern and magnitude of balance reactions in humans. J Vestib Res. 1998;8:381-97.

35. Nakamura H, Tsuchida T, Mano Y. The assessment of posture control in the elderly using the displacement of the center of pressure after forward platform translation. J Electromyogr Kinesiol. 2001;11:395-403.

36. Woo J, Hong A, Lau E, Lynn H. A randomised controlled trial of tai chi and resistance exercise on bone health, muscle strength and balance in community-living elderly people. Age Ageing. 2007;36:262-8.

37. Wayne PM, Walsh JN, Taylor-Piliae RE, Wells RE, Papp KV, Donovan NJ, et al. Effect of tai chi on cognitive performance in older adults: systematic review and meta-analysis. J Am Geriatr Soc. 2014;62:25-39.

38. Zheng G, Liu F, Li S, Huang M, Tao J, Chen L. Tai chi and the protection of cognitive ability: a systematic review of prospective studies in healthy adults. Am J Prev Med. 2015;49:89-97.

39. Miller SM, Taylor-Piliae RE. Effects of tai chi on cognitive function in community-dwelling older adults: a review. Geriatr Nurs. 2014;35:9-19.

\section{Publisher's Note}

Springer Nature remains neutral with regard to jurisdictional claims in published maps and institutional affiliations.

Ready to submit your research? Choose BMC and benefit from:

- fast, convenient online submission

- thorough peer review by experienced researchers in your field

- rapid publication on acceptance

- support for research data, including large and complex data types

- gold Open Access which fosters wider collaboration and increased citations

- maximum visibility for your research: over $100 \mathrm{M}$ website views per year

At BMC, research is always in progress.

Learn more biomedcentral.com/submissions 